\title{
Low-frequency high-resolution radio observations of the TeV-emitting blazar SHBL J001355.9-185406
}

\author{
Natalia Żywucka ${ }^{1}$, Arti Goyal ${ }^{1}$, Marek Jamrozy ${ }^{1}$, Michał Ostrowski ${ }^{1}$, and Łukasz Stawarz ${ }^{2,1}$ \\ 1 Astronomical Observatory, Jagiellonian University, ul. Orla 171, 30-244 Kraków, Poland \\ e-mail: natti@oa.uj .edu.pl \\ 2 Institute of Space and Astronautical Science JAXA, 3-1-1 Yoshinodai, Chuo-ku, Sagamihara, 252-5210 Kanagawa, Japan
}

Received 24 January 2014 / Accepted 4 February 2014

\begin{abstract}
Context. In the framework of the unification scheme of radio-loud active galactic nuclei, BL Lac objects and quasars are the beamed end-on counterparts of low-power (FR I) and high-power (FR II) radio galaxies, respectively.

Aims. Some BL Lacs have been found to possess the FR II-type, large-scale radio morphology, suggesting that the parent population of BL Lacs is a mixture of low- and high-power radio galaxies. This seems to apply only to "low frequency-peaked" BL Lacs, since all the "high frequency-peaked" BL Lacs studied so far were shown to host the FR I-type radio jets, exclusively. While analyzing the NRAO VLA Sky Survey (NVSS) maps of the TeV detected BL Lacs, we have discovered, however, that the high frequency-peaked object SHBL J001355.9-185406 is associated uniquely with the one-sided, arcmin-scale, and edge-brightened jet/lobe-like feature extending to the south-west from the blazar core.

Methods. In order to investigate the large-scale morphology of SHBL J001355.9-185406 in detail, we have performed low-frequency and high-resolution observations of the source at 156, 259, and $629 \mathrm{MHz}$ using the Giant Metrewave Radio Telescope (GMRT).

Results. Our analysis indicates that no diffuse arcmin-scale emission is present around the unresolved blazar core, and that the arcmin-scale structure seen on the NVSS map breaks into three distinct features unrelated to the blazar, but instead associated with background AGN.

Conclusions. The upper limits for the extended radio halo around the TeV-emitting BL Lac object SHBL J001355.9-185406 read as $<10 \%-1 \%$ at $156-629 \mathrm{MHz}$. The fact that the integrated radio spectrum of the unresolved blazar core is flat down to $156 \mathrm{MHz}$ indicates that a self-similar character of the jet in the source holds up to relatively large distances from the jet base.
\end{abstract}

Key words. BL Lacertae objects: individual: SHBL J001355.9-185406 - radio continuum: galaxies

\section{Introduction}

Blazars constitute the most extreme class of active galactic nuclei (AGN), for which the total radiative output is dominated by a Doppler-boosted and highly variable emission of a nuclear relativistic jet observed at a small viewing angle. The blazar family includes flat-spectrum radio-loud quasars (FSRQs) and BL Lacertae (BL Lac) objects. In the framework of the "unified scheme" of AGN, powerful radio galaxies of the Fanaroff-Riley morphological type II (FR IIs; see, Fanaroff \& Riley 1974) constitute the parent population of FSRQs, while low-power radio galaxies of the Fanaroff-Riley morphological type I (FR Is) are considered to be "misaligned" BL Lacs (Barthel 1989; Urry \& Padovani 1995; Xu et al. 2009).

High dynamic range radio imaging of BL Lac objects at $\mathrm{GHz}$ frequencies often reveals the presence of a diffuse extended radio emission, with the integrated luminosity exceeding, in several cases, the FR I/FR II divide. We note that this borderline scales with the optical luminosity of the host as $\propto L_{\mathrm{opt}}^{1.8}$, with the normalization $L_{1.4 \mathrm{GHz}}=10^{24} \mathrm{~W} \mathrm{~Hz}^{-1}$ for the absolute isophotal magnitude of the host galaxy -21 measured to 24.5 mag per $\operatorname{arcsec}^{2}$ in the rest-frame of a source (Ledlow \& Owen 1996). This may indicate that the parent population of BL Lac objects is a mixture of FR I- and FR II-type galaxies (see, e.g., Cassaro et al. 1999; Rector \& Stocke 2001; Landt \& Bignall 2008; Kharb et al. 2010). Therefore, a careful investigation of the extended diffuse radio emission in BL Lacs is of importance for models relating the AGN accretion power and jet luminosity, in general, and blazar population studies, in particular (e.g., Blandford \& Payne 1982; Rawlings \& Saunders 1991; Ghisellini et al. 2011).

The high frequency-peaked BL Lacs (HBLs), i.e., blazars with synchrotron peak frequencies $>10^{15} \mathrm{~Hz}$ (see the discussion in Abdo et al. 2010, and references therein), dominate the population of extragalactic $\mathrm{TeV}$ emitters ${ }^{1}$. These sources, unlike the low frequency-peaked BL Lacs (LBLs; synchrotron peak frequencies $<10^{15} \mathrm{~Hz}$ ), seem to be associated strictly with FR Itype large-scale radio structures (e.g., Kharb et al. 2010) and they form a relatively distinct sub-class of blazars with respect to jet physical properties (e.g., Tavecchio et al. 2010). Also, no superluminal velocities have been detected in the TeV-emitting HBLs on milli-arcsec scales until now, despite several dedicated observational programs (e.g., Piner et al. 2010, and references therein). Such apparent superluminal velocities are typical for FSRQs and not rare for LBLs.

The blazar SHBL J001355.9-185406 (J2000.0 RA = $00^{\mathrm{h}} 13^{\mathrm{m}} 56^{\mathrm{s}} .054$, Dec $=-18^{\circ} 54^{\prime} 06^{\prime \prime} .48$; redshift $z=0.0948$ according to Jones et al. 2009) is a peculiar example of an HBL. This source was listed in a catalog of extremely high X-rayto-radio flux ratio targets in the multi-frequency "Sedentary Survey" of HBLs performed by Giommi et al. (2005).

\footnotetext{
http://tevcat.uchicago.edu/
} 
Table 1. Observational summary.

\begin{tabular}{|c|c|c|c|c|c|c|c|c|c|c|c|}
\hline $\begin{array}{l}\nu_{\mathrm{RF}} \\
(\mathrm{MHz}) \\
(1)\end{array}$ & $\begin{array}{c}B W \\
(\mathrm{MHz}) \\
(2)\end{array}$ & $\begin{array}{c}\text { Date } \\
\text { (dd.mm.yy) } \\
\text { (3) }\end{array}$ & $\begin{array}{l}\text { Time } \\
(\mathrm{hr}) \\
(4)\end{array}$ & $\begin{array}{l}\text { Pol. } \\
\text { (5) }\end{array}$ & $\begin{array}{l}\text { Flux cal. } \\
\text { name (flux) } \\
\text { (6) }\end{array}$ & $\begin{array}{l}\text { Phase cal. } \\
\text { name (flux) } \\
\text { (7) }\end{array}$ & $\begin{array}{c}H P B W \\
(\operatorname{arcmin}) \\
(8)\end{array}$ & $\begin{array}{l}\text { Synth. beam } \\
\left(\operatorname{arcsec}^{2}\right) \\
(9)\end{array}$ & $\begin{array}{c}\text { PA } \\
\text { (deg) } \\
(10)\end{array}$ & $\begin{array}{c}\sigma_{\mathrm{map}} \\
(\mathrm{mJy} / \mathrm{b}) \\
(11)\end{array}$ & $\begin{array}{c}\text { Dyn. range } \\
\text { (peak/rms err.) } \\
(12)\end{array}$ \\
\hline 156 & 16 & 29.08 .11 & $\sim 6$ & RR, LL & $3 \mathrm{C} 48(65)$ & $\mathrm{J} 0025-260(20)$ & 186 & $20.3 \times 13.0$ & 23.3 & 2.0 & $\sim 31$ \\
\hline $259^{*}$ & 6.5 & 01.09 .11 & $\sim 6$ & LL & $3 \mathrm{C} 48(47)$ & $\mathrm{J} 0025-260(18)$ & 116 & $15.1 \times 10.5$ & 37.4 & 0.09 & $\sim 60$ \\
\hline $629^{*}$ & 32 & 01.09 .11 & $\sim 6$ & $\mathrm{RR}$ & $3 \mathrm{C} 48(30)$ & J0025-260(14) & 45 & $6.4 \times 4.1$ & 32.9 & 0.07 & $\sim 600$ \\
\hline 8434 & 50 & 06.06 .98 & $\sim 0.08$ & $\mathrm{RR}, \mathrm{LL}$ & $3 \mathrm{C} 48(3.3)$ & J0110-076(0.55) & 6 & $0.48 \times 0.28$ & -31.07 & 0.09 & $\sim 150$ \\
\hline
\end{tabular}

Notes. Columns: (1) central radio frequency $v_{\mathrm{RF}}$ of observation $\left(^{(*)}\right.$ indicates a dual frequency mode; ${ }^{(\dagger)}$ archival VLA data obtained in AnB configuration in 2 IF); (2) total band-width (BW) of observation; (3) date of observation; (4) time spent on the source excluding calibration overheads; (5) polarization; (6) the flux calibrator used in the observation (flux density of the calibrator in Jy); (7) the phase calibrator used during the observation (flux density of the calibrator in Jy); (8) half power beam width $(H P B W=1.22 \cdot \lambda / D$; where $\lambda$ is the wavelength of observation and $D$ is an antenna diameter); (9) synthesized beam achieved; (10) position angle (PA) of the restoring beam; (11) rms error in the map; (12) dynamic range achieved.

Its $0.1-2.4 \mathrm{keV} \mathrm{X}$-ray flux is $1.26 \times 10^{-11} \mathrm{erg} \mathrm{s}^{-1} \mathrm{~cm}^{-2}$. The corresponding $1400 \mathrm{MHz}$ radio flux spectral density is $29.2 \pm$ $1.0 \mathrm{mJy}$ (see, Condon et al. 1998). This source has been detected very recently at the level of $\sim 1 \%$ of the flux of the Crab nebula above $300 \mathrm{GeV}$ by the High Energy Stereoscopic System (H.E.S.S.; Abramowski et al. 2013). Analysis of the Fermi Large Area Telescope (Fermi-LAT) data revealed a faint and flatspectrum counterpart of SHBL J001355.9-185406 at the GeV photon energies, with an integrated photon flux above $100 \mathrm{MeV}$ of $(0.9 \pm 0.7) \times 10^{9} \mathrm{ph} \mathrm{cm}^{-2} \mathrm{~s}^{-1}$ and a photon index of $1.5 \pm 0.2$ (Sanchez \& Fegan 2010). At lower frequencies, the discussed object was also reported as a bright and variable NIR/optical emitter. In particular, the NIR flux of SHBL J001355.9-185406 determined in 2010 was about 0.7 mag brighter than that previously published in the Two Micron All Sky Survey (2MASS; Carrasco et al. 2010). The corresponding 70\% flux increase suggests a jet origin for the NIR continuum.

The peculiarity of the SHBL J001355.9-185406 is related, however, to its large-scale radio structure, which was found when examining the archival radio National Radio Astronomy Observatory (NRAO) Very Large Array (VLA) Sky Survey (NVSS; Condon et al. 1998) maps of the TeV-detected BL Lacs. This structure, shown in Fig. 1, consists of a compact core component, as expected for a blazar, but also of a one-sided and elongated lobe/jet-like feature extending to the south-west from the nucleus. At the redshift of the source (luminosity distance of $d_{L}=430 \mathrm{Mpc}$ for the flat cosmology with $H_{0}=$ $71 \mathrm{~km} \mathrm{~s}^{-1} \mathrm{Mpc}^{-1}, \Omega_{\mathrm{M}}=0.27$ and $\Omega_{\Lambda}=0.73$ ), the $3.3 \mathrm{ex}-$ tension of the lobe corresponds to a physical (projected) size of $\sim 344 \mathrm{kpc}$ and the $1400 \mathrm{MHz}$ flux, $15.8 \mathrm{mJy}$, corresponds to a monochromatic luminosity of $\sim 5 \times 10^{39} \mathrm{erg} \mathrm{s}^{-1}$ for the lobe. The derived extended luminosity is therefore comfortably low, as would be expected for a low-power blazar of HBL type, yet the morphology of the giant lobe is striking for two reasons.

First, the lobe is clearly edge-brightened, and hence the whole large-scale radio structure of SHBL J001355.9-185406 resembles closely an FR II-type object than an FR I-type object. Second, the extended structure in the discussed source is clearly one-sided. This is not expected for the large-scale structures in radio-loud AGN in general, regardless of their Fanaroff-Riley type, since the lobes' expansion velocities on scales of tens and hundreds of kpc are only expected to be subrelativistic. In other words, the Doppler (and time-travel) effects cannot easily be blamed for the apparent lack of the counter-lobe in SHBL J001355.9-185406 (see, however, Stawarz 2004; and Bagchi et al. 2007). In order to investigate this issue in more detail, we performed deep, high-resolution radio observations of

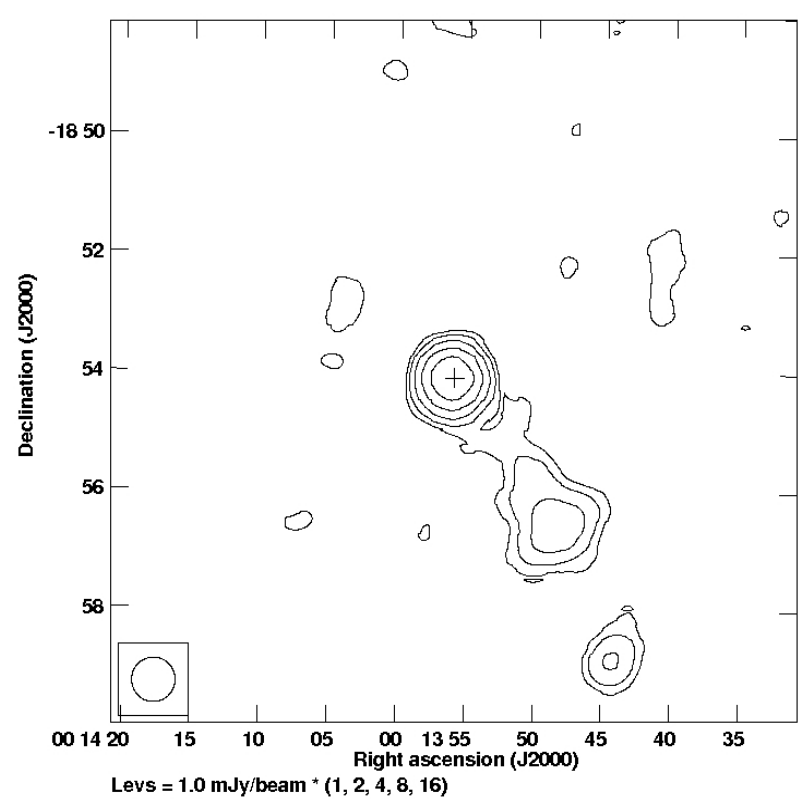

Fig. 1. Contour plot of the $1400 \mathrm{MHz}$ NVSS radio continuum emission. The plus sign marks the position of blazar core and the bottom left inset shows the size of the restoring beam.

SHBL J001355.9-185406 at low frequencies. The results of the analysis are presented below.

\section{Observations and data analysis}

Observations were carried out using the GMRT in three frequency bands, namely 156, 259, and $629 \mathrm{MHz}$, the latter two obtained in dual frequency mode (Swarup 1991). Data were recorded with an integration time of $8 \mathrm{~s}$ with the available frequency band divided into 256 channels, following the usual protocol of observing flux and phase calibrator interlaced with observation on the source. At the beginning and end of the observing run, flux calibrator 3C 48 was observed for about $15 \mathrm{~min}$. The phase calibrator J0025-260, was observed every $\sim 25 \mathrm{~min}$ for $\sim 4-5 \mathrm{~min}$. Table 1 gives the summary of the observations. The data reduction was carried out following standard calibration and reduction procedures in the Astronomical Image Processing System (AIPS). Data were edited for strong radio frequency interference (RFI) and then standard flux and phase calibration were applied to the source. In addition to the newly obtained GMRT data, we have also analyzed the 
archival $8434 \mathrm{MHz}$ VLA data for SHBL J001355.9-185406 in a standard fashion as described below.

The Baars et al. (1977) absolute flux density scale was used to determine flux densities of the flux calibrators, phase calibrator, and the source. The calibrated data were channel collapsed after the bandpass calibration using the phase calibrator, and the deconvolved images were made using the task "IMAGR". To account for wide-field imaging with non-coplanar baselines, polyhedron imaging was used in "IMAGR" (Cornwell \& Perley 1992), where the field of view is subdivided into a number of smaller fields (facets). These were $5^{\prime} \times 5^{\prime}, 5^{\prime} \times 5^{\prime}$, and $7^{\prime} \times 7^{\prime}$ at 629,259 , and $156 \mathrm{MHz}$, respectively, facets covering the primary beam up to the half-power beam width (HPBW). Several rounds of phase-based self-calibration were performed iteratively, by choosing point sources such that the flux density within one synthesized beam is more than $5 \sigma$. Final maps were made from the full $(u, v)$ coverage and the UV-data were weighted using Briggs robust weighting of 0 (Briggs 1995). The final images were then combined to reconstruct the sky using the task "FLATN" and the "FLATNed" image was then corrected for the primary beam ${ }^{2}$ of the antenna using the task "PBCOR".

The uncertainties in the estimated flux densities of the source (obtained using "TVSTAT" task) depend on the rms noise in the map as well as on the errors associated with uncalibrated system temperature $\left(T_{\text {sys }}\right)$ variations. These were taken to be $5 \%$ at 629 and $259 \mathrm{MHz}$, and $8 \%$ at $156 \mathrm{MHz}$. The final error on the flux density was calculated using the expression:

$\delta S=\sqrt{\left(S \times \sigma_{T_{\text {sys }}}\right)^{2}+\left(\sigma_{\text {map }} \times \sqrt{\frac{\text { area }}{\text { beam }}}\right)^{2}}$

where $S$ is the integrated flux density of the source, $\sigma_{T_{\mathrm{sys}}}$ is the rms error due to the uncalibrated system temperature, $\sigma_{\text {map }}$ is the rms error in the map, area is the area of source in pixels, and beam is the beam area in pixels.

The expected thermal noise values are about 0.1, 0.09, and $0.02 \mathrm{mJy} /$ beam at 156,259 , and $629 \mathrm{MHz}$, respectively, for our observational set-up (Table 1 ). However, the rms noise values achieved are about 20,10, and 5 times worse, as a considerable amount of the GMRT bad data was flagged during the reduction process. For the VLA observations, the rms noise is close to the expected thermal noise $\sim 0.08 \mathrm{mJy} /$ beam (Table 1).

\section{Results}

The spectral index (defined here as $S_{v} \propto v^{\alpha}$ ) for the unresolved core of SHBL J001355.9-185406 has been evaluated using the peak flux values of the blazar obtained using the task "JMFIT" from the 156, 259, and 629 MHz GMRT maps, along with the archival $352 \mathrm{MHz}$ data from the Westerbork In the Southern Hemisphere (WISH) survey (De Breuck et al. 2002), the $1400 \mathrm{MHz}$ (NVSS survey) data, and the VLA data at $8434 \mathrm{MHz}$. By applying the weighted linear regression analysis, we find $\alpha=-0.43 \pm 0.05$ (see Fig. 2). The low-frequency radio spectrum seems to be even flatter than that, with $\alpha_{156}^{629}=$ $-0.1 \pm 0.1$ (see Table 2), as indeed expected for a BL Lac object (e.g., Angel \& Stockman 1980; Cavallotti et al. 2004).

The full-resolution GMRT maps of the entire field of interest are presented in Figs. 3-5. As shown, the lobe-like structure seen to the south-west from the blazar core in the NVSS image breaks into three distinct features, marked as Source 1, Source 2,

\footnotetext{
2 http://ncra.tifr.res.in/ngk/primarybeam/beam.html
}

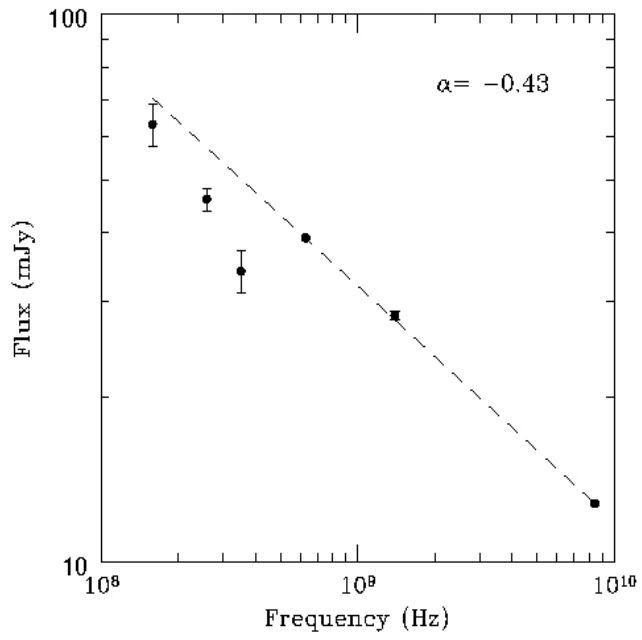

Fig. 2. Integrated radio spectrum of the unresolved core in SHBL J001355.9-185406. The dashed line shows the result of weighted linear regression analysis.

Table 2. Integrated radio flux densities obtained using "TVSTAT" in $\mathrm{mJy}$ and $156-629 \mathrm{MHz}$ spectral indices.

\begin{tabular}{lccccc}
\hline \hline Source & $\begin{array}{c}156 \mathrm{MHz} \\
\text { flux (err.) }\end{array}$ & $\begin{array}{c}259 \mathrm{MHz} \\
\text { flux (err.) }\end{array}$ & $\begin{array}{c}629 \mathrm{MHz} \\
\text { flux (err.) }\end{array}$ & $\begin{array}{c}8434 \mathrm{MHz} \\
\text { flux (err.) }\end{array}$ & $\alpha_{156}^{629}$ \\
$(1)$ & $(2)$ & $(3)$ & $(4)$ & $(5)$ & $(6)$ \\
\hline Blazar & $55(6.0)$ & $53(3.7)$ & $45(2.2)$ & $15(2.3)$ & $-0.1(0.1)$ \\
Source 1 & $29(5.3)$ & $5(1.6)$ & $4.0(0.3)$ & - & $-1.4(0.2)$ \\
Source 2 & $62(7.5)$ & $47(4.0)$ & $17(1.0)$ & - & $-0.9(0.1)$ \\
Source 3 & $11(3.4)$ & $15(2.4)$ & $5.8(0.4)$ & - & $-0.5(0.3)$ \\
\hline
\end{tabular}

Notes. Columns: (1) source name; (2)-(5) integrated radio flux densities and the errors in parentheses at $156 \mathrm{MHz}, 259 \mathrm{MHz}, 629 \mathrm{MHz}$, and $8434 \mathrm{MHz}$; (6) integrated spectral indices between $156-629 \mathrm{MHz}$ for the sources mentioned in the text.

and Source 3 in Fig. 3. Table 2 gives the integrated flux densities for the unresolved blazar core and the above mentioned sources. Detailed morphologies of all the detected objects are best displayed in the $629 \mathrm{MHz}$ map, which is the best quality map out of the three obtained GMRT maps (Table 1); the corresponding Fig. 5 also includes the low-resolution $1400 \mathrm{MHz}$ contours for comparison, as well as the overlaid Digitized Sky Survey (DSS $R$-band) image. We note that in the $8434 \mathrm{MHz}$ map, only the point-like blazar is detected, hence the corresponding image is not presented here.

Figure 6 shows the spectral index map between 156 and $629 \mathrm{MHz}$ frequencies. When generating this map, the $629 \mathrm{MHz}$ images were made with $13 k \lambda$ uvrange (equal to the UVrange obtained at $156 \mathrm{MHz}$ with the GMRT). This ensured the resultant synthesized beam in the $629 \mathrm{MHz}$ map closely resembling the beam at $156 \mathrm{MHz}$, although the two beams are not exactly equal due to the dependence on the filling of the visibility plane (e.g., Cornwell et al. 1999). Next, the two maps were convolved to the common circular beam equal to the major axis beam at $156 \mathrm{MHz}$ (i.e., 21"). As shown in the resulting figure, and already mentioned above, the unresolved blazar core displays a flat spectrum with $\alpha_{156}^{629} \simeq 0$; the three newly resolved structures to the south-west are, however, characterized by steep spectra, $\alpha_{156}^{629}<-0.5$ (see Table 2). 


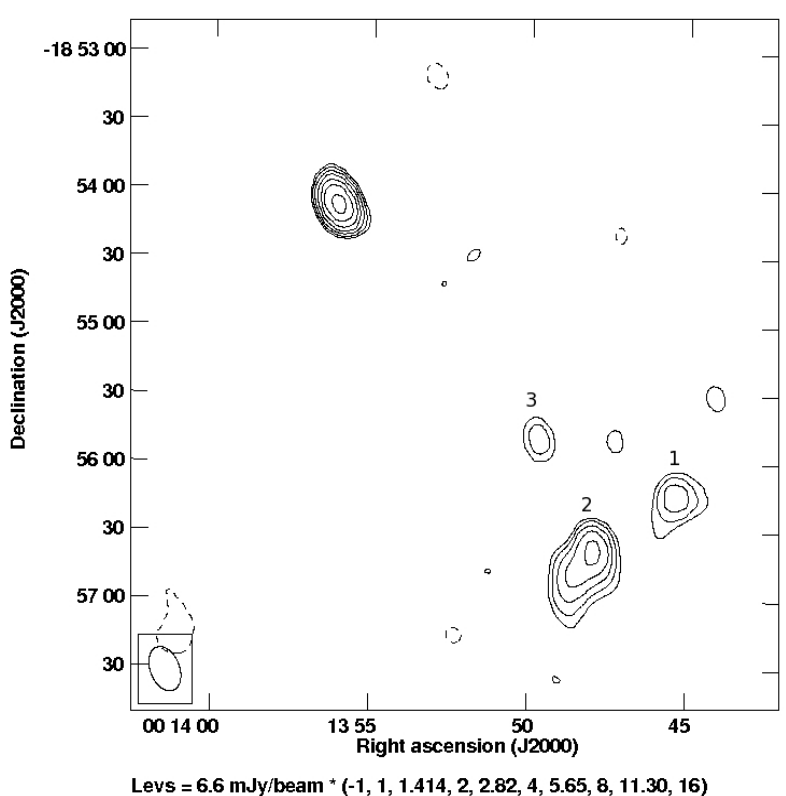

Fig. 3. A $156 \mathrm{MHz}$ GMRT contour map. The bright point-like source in the north-east corner of the image corresponds to the blazar core of SHBL J001355.9-185406. The newly resolved radio features to the south-west of the blazars are indicated with numbers 1, 2, and 3 .

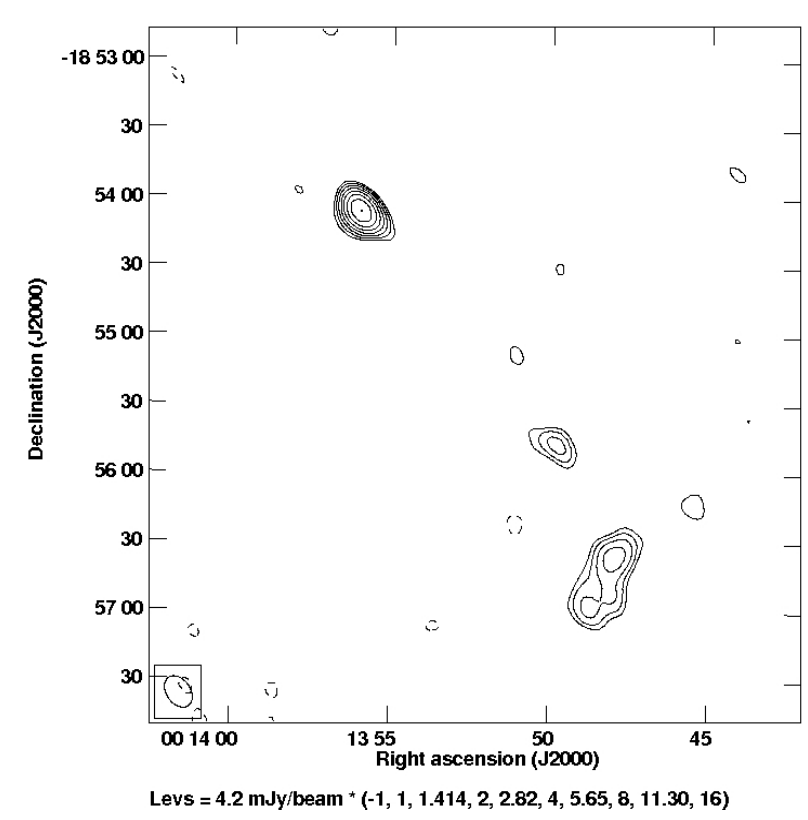

Fig. 4. A $259 \mathrm{MHz}$ GMRT contour map. The bright point-like source in the north-east corner of the image corresponds to the blazar core of SHBL J001355.9-185406.

The GMRT has a hybrid configuration, with 14 out of 30 antennas located in a $1 \mathrm{~km}$ square area (known as the central square). The typical antenna spacing available in the central square with the GMRT is about $\sim 800 \mathrm{~m}$. This assures sensitivity to sources with angular sizes up to $\sim 8^{\prime}, \sim 5^{\prime}$, and $\sim 2$ '.2 at 156,259 , and $629 \mathrm{MHz}$, respectively, even with the snapshot observations. The primary beam at 156,259 , and $629 \mathrm{MHz}$ is about $186^{\prime}, 116^{\prime}$, and $45^{\prime}$, respectively (Table 1 ). Since the extension of the lobe-like structure to the south-west from the blazar core is about $3 ! 3$, the entire feature should be well within the primary beam at each frequency, and the availability of multiple short spacing in our observations ensures more robust measurements of the extended flux. Therefore, we do not expect any missing flux in our observations.

We note, however, that the non-detection of the extended emission, close to the blazar core, as seen in the NVSS map (Fig. 1), could be due to the limited sensitivity achieved in our observations (see, Table 1). To investigate this issue in more detail, we computed the $1400 \mathrm{MHz}$ expected flux density per $5^{\prime \prime}$ beam (as this is the typical synthesized beam achieved at $629 \mathrm{MHz}$ using the GMRT) near the blazar core. Assuming a uniform flux density in the NVSS map in this region, we next evaluated the flux density per beam at $629 \mathrm{MHz}$ assuming the spectral index -1.0 . The extrapolated flux density per beam at $629 \mathrm{MHz}$ comes out to be $0.03 \mathrm{mJy}$, much below the rms error achieved in our observations. Hence, it is possible that the extended structure seen in the NVSS map close to the blazar core is real, but only below the sensitivity limit of our highresolution maps at low frequencies. Still, the radio morphologies of the sources detected to the south-west of the blazar implies that the brightest part of the large-scale NVSS structure is unrelated to the blazar. Source 1 can be easiliy classified as an ultrasteep spectrum source (e.g., De Breuck et al. 2000, see Table 2, Fig. 6) while Source 2 has a typical FR II radio morphology (e.g., Miley 1980; Hardcastle 2008). Source 3 breaks into two pointlike sources, each with an optical counterpart (see Fig. 5). This strongly suggests that the detected features are related to distant radio galaxies, although the lack of any obvious optical counterparts (for Sources 1 and 2), or optical spectra (Source 3) precludes us from making any definite statements in this respect.

\section{Conclusions}

In our study presented here, we investigated in detail the large-scale radio morphology of the TeV-detected blazar SHBL J001356-185406 which, on the low-resolution NVSS map, appears to be of the FR II-type. High-sensitivity and high dynamic range (Table 1) observations (which do not suffer from a missing flux; see Sect. 3) were made using the GMRT at low frequencies of 156,259 , and $629 \mathrm{MHz}$. Our analysis indicated that: (1) the integrated radio spectral index is flat down to $156 \mathrm{MHz}$ for the blazar core (Fig. 2); (2) no diffuse arcmin-scale emission is present around the unresolved blazar core (see Figs. 3-5); (3) the 3!3-long, lobe/jet-like structure extending to the southwest of the blazar core on the NVSS map breaks into three distinct, steep-spectrum radio features, unrelated to the blazar and instead most likely associated with background AGN.

As a corollary to this study, we point out that the observations presented here are rather unique, since very low-frequency, high-resolution, and high dynamic range studies of $\mathrm{TeV}$ detected HBLs are particularly sparse. The new data allow us to constrain the upper limits for the extended radio halo around the blazar as $<12 \%$ at $156 \mathrm{MHz},<8 \%$ at $259 \mathrm{MHz}$, and $<0.9 \%$ at $629 \mathrm{MHz}$ of the core emission. This is in agreement with the early VLA studies of large-scale structures of BL Lacs at GHz frequencies by Ulvestad \& Johnston (1984) and Ulvestad \& Antonucci (1986), who found that only a few of the objects from the analyzed samples possess any arcminute-scale structures with total fluxes exceeding $\sim 0.1 \%$ of the core emission.

The another interesting result of our study is that the radio spectral index of the SHBL J001356-185406 core remains flat down to $156 \mathrm{MHz}$, with no dramatic curvature. Flat spectra (indices $\alpha \sim 0$ ) of relativistic jets observed at small viewing 


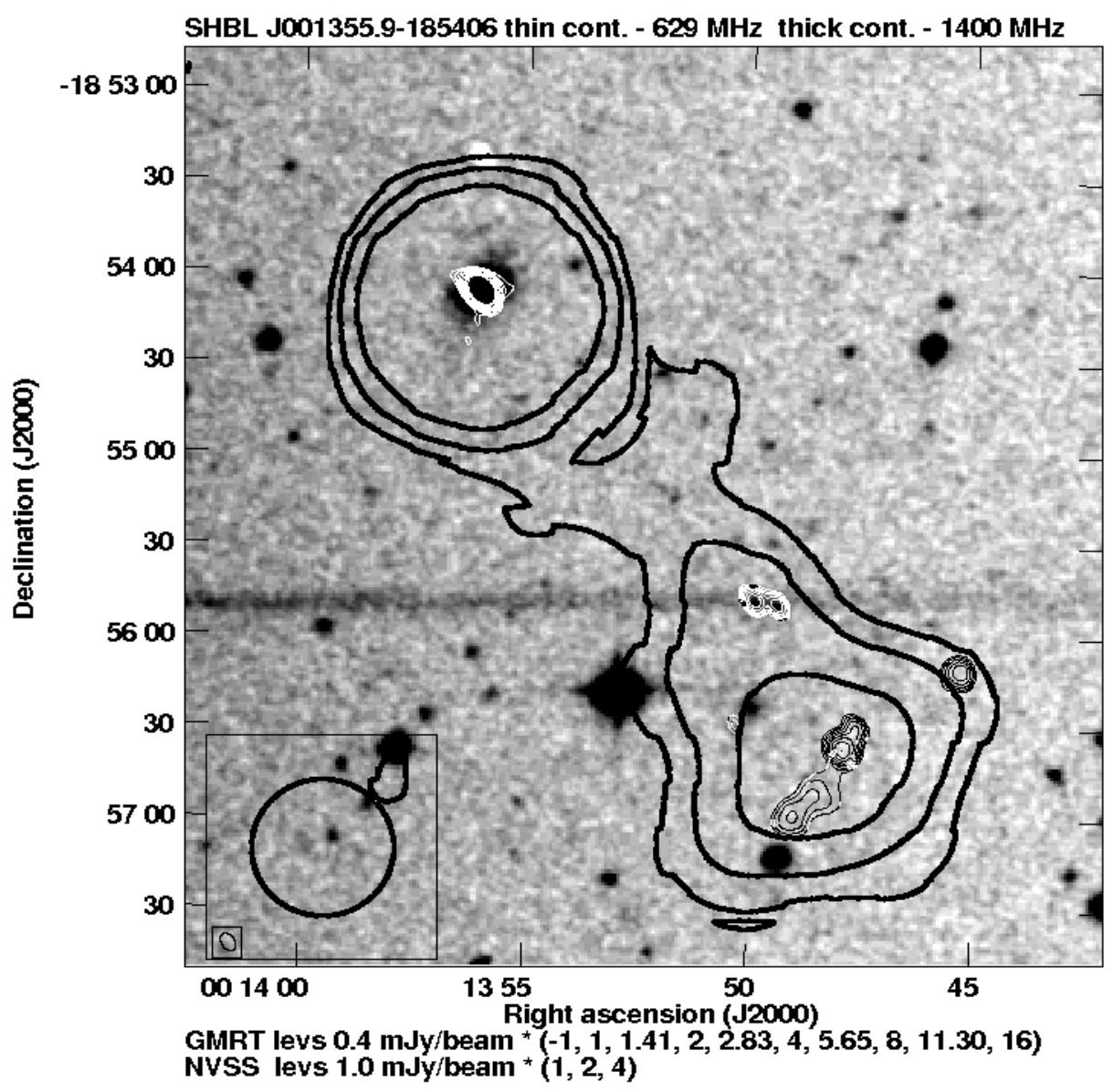

Fig. 5. A $629 \mathrm{MHz}$ GMRT contour map (thin cont.). The bright point-like source in the north-east corner of the image corresponds to the blazar core of SHBL J001355.9-185406. The $1400 \mathrm{MHz}$ NVSS map is represented by the thick contours, which are overlaid on the DSS $R$-band image. The bottom-left inset image shows the synthesized beams at $629 \mathrm{MHz}$ (thin cont.) and $1400 \mathrm{MHz}$ (thick cont.).

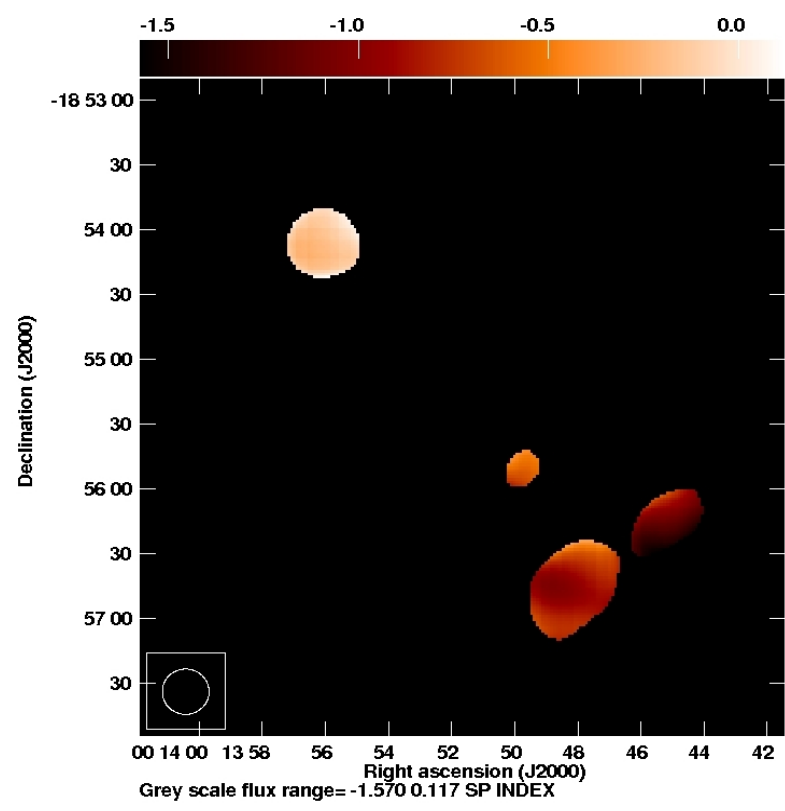

Fig. 6. The $156-629 \mathrm{MHz}$ spectral index distribution. Both 156 and $629 \mathrm{MHz}$ maps were convolved to a common circular beam of $21^{\prime \prime} \times 21^{\prime \prime}$

angles are widely believed to arise due to a superposition of selfabsorbed spectra of different parts of an outflow with conserved magnetic flux and maintained power-law spectrum of the emitting electrons (Blandford \& Königl 1979). The fact that in the case of the SHBL J001356-185406 core we do not see any spectral turnover down to $156 \mathrm{MHz}$ indicates, therefore, that a self-similar character of the jet in the source holds up to relatively large distances from the jet base.

Acknowledgements. We thank the anonymous referee for his/her helpful suggestions. We thank the staff of the GMRT that made these observations possible. GMRT is run by the National Centre for Radio Astrophysics of the Tata Institute of Fundamental Research. This research has made use of NASA/IPAC Extragalactic Database (NED), which is operated by the Jet Propulsion Laboratory, California Institute of Technology, under contract with National Aeronautics and Space Administration. The National Radio Astronomy Observatory is a facility of the National Science Foundation operated under cooperative agreement by Associated Universities, Inc. This work was supported by the Polish National Science Centre through the grant DEC2012/04/A/ST9/00083. M.J. is supported by Polish National Science Center grant DEC-2013/09/B/ST9/00599.

\section{References}

Abdo, A. A., Ackermann, M., Agudo, I., et al. 2010, ApJ, 716, 30

Abramowski, A., Acero, F., Aharonian, F., et al. 2013, A\&A, 554, A72

Angel, J. R. P., \& Stockman, H. S. 1980, ARA\&A, 18, 321

Baars, J. W. M., Genzel, R., Pauliny-Toth, I. I. K., \& Witzel, A. 1977, A\&A, 61, 99

Bagchi, J., Gopal-Krishna, Krause, M., \& Joshi, S. 2007, ApJ, 670, L85

Barthel, P. D. 1989, ApJ, 336, 606 
Blandford, R. D., \& Königl, A. 1979, ApJ, 232, 34

Blandford, R. D., \& Payne, D. G. 1982, MNRAS, 199, 883

Briggs, D. S. 1995, Ph.D. Thesis, Socorro, NM: New Mexico Institute of Mining Technology

Carrasco, L., Carramiñana, A., Recillas, E., et al. 2010, ATel, 3023,

Cassaro, P., Stanghellini, C., Bondi, M., et al. 1999, A\&AS, 139, 60

Cavallotti, F., Wolter, A., Stocke, J. T., \& Rector, T. 2004, A\&A, 419, 459

Condon, J. J., Cotton, W. D., Greisen, E. W., et al. 1998, AJ, 115, 1693

Cornwell, T. J., \& Perley, R. A. 1992, A\&A, 261, 353

Cornwell, T., Braun, R., \& Briggs, D. S. 1999, in Synthesis Imaging in Radio Astronomy II, eds. G. B. Taylor, C. L. Carilli, \& R. A. Perley, ASP Conf. Ser., 180,151

De Breuck, C., van Breugel, W., Röttgering, H. J. A., \& Miley, G. 2000, A\&AS, 143,303

De Breuck, C., Tang, Y., de Bruyn, A. G., Rottgering, H., \& van Breugel, W. 2002, VizieR Online Data Catalog: VIII/69

Fanaroff, B. L., \& Riley, J. M. 1974, MNRAS, 167, 31

Ghisellini, G., Tavecchio, F., Foschini, L., \& Ghirlanda, G. 2011, MNRAS, 414 2674

Giommi, P., Piranomonte, S., Perri, M., \& Padovani, P. 2005, A\&A, 434 385
Hardcastle, M. J. 2008, in Extragalactic Jets: Theory and Observation from Radio to Gamma Ray, eds. T. A. Rector, \& D. S. De Young, ASP Conf. Ser., 386,46

Jones, D. H., Read, M. A., Saunders, W., et al. 2009, MNRAS, 399, 683

Kharb, P., Lister, M. L., \& Cooper, N. J. 2010, ApJ, 710, 764

Landt, H., \& Bignall, H. E. 2008, MNRAS, 391, 967

Ledlow, M. J., \& Owen, F. N. 1996, AJ, 112, 9

Miley, G. 1980, ARA\&A, 18, 165

Piner, B. G., Pant, N., \& Edwards, P. G. 2010, ApJ, 723, 1150

Rawlings, S., \& Saunders, R. 1991, Nature, 349, 138

Rector, T. A., \& Stocke, J. T. 2001, AJ, 122, 565

Sanchez, D., \& Fegan, S. 2010, ATel, 3014,

Stawarz, Ł. 2004, ApJ, 613, 119

Swarup, G. 1991, in Radio Interferometry. Theory, Techniques, and Applications, eds. T. J. Cornwell, \& R. A. Perley, IAU Colloq. 131, ASP Conf. Ser., 19, 376

Tavecchio, F., Ghisellini, G., Ghirlanda, G., Foschini, L., \& Maraschi, L. 2010, MNRAS, 401, 1570

Ulvestad, J. S., \& Antonucci, R. R. J. 1986, AJ, 92, 6

Ulvestad, J. S., \& Johnston, K. J. 1984, AJ, 89, 189

Urry, C. M., \& Padovani, P. 1995, PASP, 107, 803

Xu, Y.-D., Cao, X., \& Wu, Q. 2009, ApJ, 694, L107 